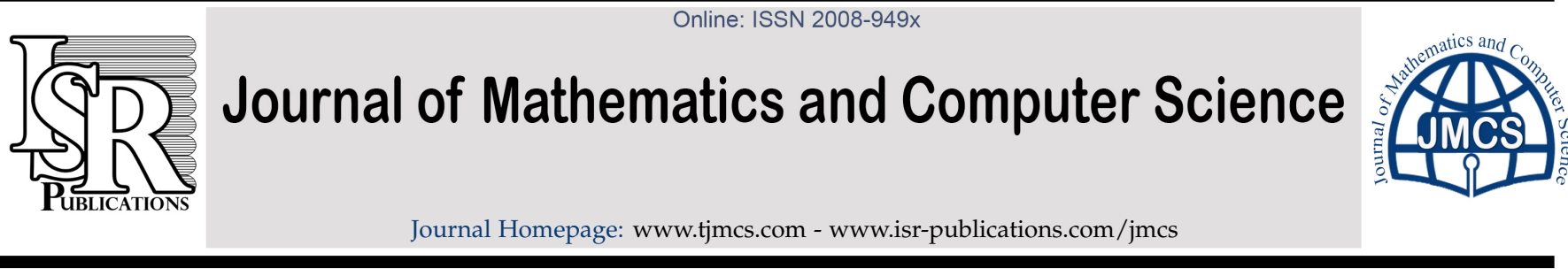

\title{
On the inclusion graphs of S-acts
}

\author{
Abdolhossein Delfan, Hamid Rasouli*, Abolfazl Tehranian \\ Department of Mathematics, Science and Research Branch, Islamic Azad University, Tehran, Iran.
}

\begin{abstract}
In this paper, we define the inclusion graph $\operatorname{Im} \mathbb{C}(A)$ of an $S$-act $A$ which is a graph whose vertices are non-trivial subacts of $A$ and two distinct vertices $B_{1}, B_{2}$ are adjacent if $B_{1} \subset B_{2}$ or $B_{2} \subset B_{1}$. We investigate the relationship between the algebraic properties of an $S$-act $A$ and the properties of the graph $\mathbb{I m \mathbb { C }}(A)$. Some properties of $\mathbb{I m \mathbb { C }}(A)$ including girth, diameter and connectivity are studied. We characterize some classes of graphs which are the inclusion graphs of S-acts. Finally, some results concerning the domination number of such graphs are given.
\end{abstract}

Keywords: S-Act, inclusion graph, diameter, girth, domination number.

2010 MSC: 20M30, 16W22, 05C12, 05C69.

(C)2018 All rights reserved.

\section{Introduction and preliminaries}

The notion of an S-act over a monoid $S$ is a fundamental concept in algebra, theoretical computer science and a variety of applications like automata theory and mathematical linguistics. Assigning graphs to algebraic structures is an approach to study algebraic properties via graph-theoretic properties. In this direction, many authors, e.g. [2, 3, 4, 7, 11, 12, 14], have been performed in connecting graph structures to various algebraic objects. Recently, inclusion graphs attached to rings, vector spaces and groups have been studied in $[1,8,5]$. Moreover, some works associating graphs to $S$-acts can be found in $[6,9,13]$.

In this paper, we associate a graph $\operatorname{Imc}(A)$ to an $S$-act $A$, called the inclusion graph of $A$, whose vertices are non-trivial subacts of $A$ in such a way that two distinct vertices $B_{1}, B_{2}$ are adjacent if $B_{1} \subset B_{2}$ or $B_{2} \subset B_{1}$. We investigate the relationship between the algebraic properties of an $S$-act $A$ and the properties of the graph $\operatorname{Im} \mathbb{C}(A)$. First we determine the girth and diameter of $\operatorname{Im} \mathbb{C}(A)$. Then some classes of graphs which are the inclusion graphs of S-acts are characterized. Finally, we present some results dealing with the domination number of such graphs.

The following is a brief account of some basic definitions about S-acts and graphs.

\footnotetext{
*Corresponding author

Email addresses: a.delfan@khoiau.ac.ir (Abdolhossein Delfan), hrasouli@srbiau.ac.ir (Hamid Rasouli), tehranian@srbiau.ac.ir (Abolfazl Tehranian)
}

doi: $10.22436 /$ jmcs.018.03.10

Received: 2017-11-23 Revised: 2018-01-10 Accepted: 2018-01-30 
Throughout this paper, unless otherwise stated, $S$ denotes a monoid with the identity 1 . By a (left) $S$-act, we mean a non-empty set $A$ on which $S$ acts unitarily, that is, $(s t) a=s(t a)$ and $1 a=a$ for all $s, t \in S$ and $a \in A$. A (non-empty proper) subset $B$ of $A$ is called a (non-trivial) subact of $A$ if $s b \in B$ for every $s \in S, b \in B$. The set of all non-trivial subacts of $A$ is denoted by $\operatorname{Sub}(A)$. A non-empty subset $I$ of $S$ is said to be a left ideal of $S$ if $s t \in I$ for any $s \in S, t \in I$. Considering $S$ as an $S$-act, any left ideal of $S$ is a subact of $S$. An element $\theta \in A$ is said to be a zero element, if $s \theta=\theta$ for all $s \in S$. A simple $S$-act is the one with no non-trivial subact. A completely reducible $S$-act is one which is a disjoint union of simple subacts. For more information about $S$-acts and related notions, the reader is referred to [10].

Let $G$ be a (simple) graph with a vertex set $V(G)$. By order of $G$, we mean the cardinality of $V(G)$ which is simply denoted by $|G|$. For any $u, v \in V(G)$, a $u, v$-path (or $u-v$ ) is a path with starting vertex $u$ and ending vertex $v$. The distance between two vertices $u, v$, denoted by $d(u, v)$, is defined as the length of the shortest path joining $u$ and $v$ if it exists, and otherwise, $d(u, v)=\infty$. The diameter of $G$, denoted by $\operatorname{diam}(\mathrm{G})$, is the largest distance between pairs of vertices of $G$. The number of vertices adjacent to a vertex $v$ is called the degree of $v$ and denoted by $\operatorname{deg}(v)$. The girth of a graph is the length of its shortest cycle, and a graph with no cycle has infinite girth. A null graph is a graph with no edges. A graph is connected if there is a path between every two distinct vertices. A complete graph is a graph in which every pair of distinct vertices are adjacent. We denote the complete graph with $n$ vertices by $K_{n}, n \in \mathbb{N}$. A path and a cycle of length $n$ are denoted by $P_{n}$ and $C_{n}$, respectively. Two graphs $G_{1}, G_{2}$ are isomorphic if and only if there exists a bijection from $V\left(G_{1}\right)$ to $V\left(G_{2}\right)$ preserving the adjacency and non-adjacency. For undefined terms and concepts about graphs, one may consult [15].

\section{Main results}

In this section we first determine the girth of the $\operatorname{graph} \operatorname{Im} \mathbb{C}(A)$ for an $S$-act $A$. Then we characterize those cycles which are inclusion graphs of some $S$-acts. Moreover, we study connectivity and diameter for the inclusion graphs. Finally, the domination number of such graphs is briefly studied.

Note that the inclusion graph for a simple $S$-act is undefined because it has no vertex. So we consider non-simple $S$-acts when dealing with their inclusion graphs throughout the paper.

Remark 2.1. It is clear that if $A$ and $B$ are isomorphic $S$-acts, then their graphs $\operatorname{Im} \mathbb{C}(A)$ and $\operatorname{Im} \mathbb{C}(B)$ are equivalent. The converse is not true in general. To see this, take the monoid $S=\{1, s\}$ where $s^{2}=1$. Consider two $S$-acts $A=\{a, b, c\}$ with trivial action and $B=\{a, b, c, d\}$ presented by the following action table:

\begin{tabular}{c|cccc} 
& $a$ & $b$ & $c$ & $d$ \\
\hline 1 & $a$ & $b$ & $c$ & $d$ \\
$s$ & $a$ & $b$ & $d$ & $c$
\end{tabular}

The non-trivial subacts of $A$ and $B$ are

$$
A_{1}=\{a\}, \quad A_{2}=\{a, b\}, \quad A_{3}=\{b\}, \quad A_{4}=\{b, c\}, \quad A_{5}=\{c\}, \quad A_{6}=\{a, c\},
$$

and

$$
B_{1}=\{a\}, \quad B_{2}=\{a, b\}, \quad B_{3}=\{b\}, \quad B_{4}=\{b, c, d\}, \quad B_{5}=\{c, d\}, \quad B_{6}=\{a, c, d\},
$$

respectively. Then $\operatorname{Im} \mathbb{C}(A) \cong \operatorname{Im} \mathbb{C}(B) \cong C_{6}$ whereas $A$ and $B$ are not isomorphic $S$-acts:
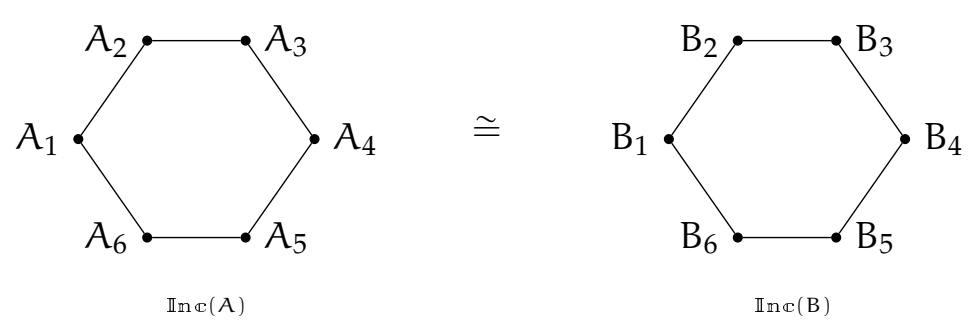
It is natural to ask whether a graph is isomorphic to the inclusion graph of an S-act. Here we consider complete graphs and cycles and characterize those ones satisfying this property.

We say that an $S$-act $A$ is uniserial, if all of its subacts are totally ordered by inclusion, or equivalently, for any two (cyclic) subacts $B$ and $C$ of $A$, either $B \subseteq C$ or $C \subseteq B$. This generalizes the well-known notion of a uniserial module extensively studied in the literature.

Clearly, for each $S$-act $A$, the graph $\operatorname{Im} \mathbb{C}(A)$ is complete if and only if $A$ is a uniserial $S$-act. The following example shows that every complete graph is the inclusion graph of a (uniserial) $S$-act. As we shall see, this is not the case for cycles in general.

\section{Example 2.2.}

(i) Consider the monogenic semigroup $S=\left\{s, s^{2}, s^{3}, \ldots, s^{n+1}\right\}, s^{n+2}=s^{n+1}, n \in \mathbb{N}$. Then all distinct non-trivial left ideals of $S$ form the chain

$$
\left\langle\mathrm{s}^{\mathrm{n}}\right\rangle \subset\left\langle\mathrm{s}^{\mathrm{n}-1}\right\rangle \subset\left\langle\mathrm{s}^{\mathrm{n}-2}\right\rangle \subset \cdots \subset\langle\mathrm{s}\rangle,
$$

where $\left\langle\mathrm{s}^{\mathrm{k}}\right\rangle=\left\{\mathrm{s}^{\mathrm{i}} \mid \mathrm{k}+1 \leqslant \mathrm{i} \leqslant \mathrm{n}+1\right\}$, for every $1 \leqslant \mathrm{k} \leqslant \mathrm{n}$. So $\mathrm{S}$ is a uniserial $\mathrm{S}$-act and clearly the graph $\operatorname{Im} \mathbb{C}(S)$ is isomorphic to the complete graph $K_{n}$. In particular, the inclusion graph of the monogenic semigroup $S=\left\{s, s^{2}, s^{3}, s^{4}\right\}, s^{5}=s^{4}$, is isomorphic to the cycle $C_{3}$ with the vertices $I_{1}=\left\{s^{4}\right\}, I_{2}=$ $\left\{s^{3}, s^{4}\right\}, I_{3}=\left\{s^{2}, s^{3}, s^{4}\right\}:$

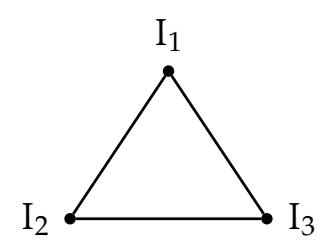

(ii) The non-trivial left ideals of the semigroup $S=(\mathbb{N},+)$ are exactly the sets $n+\mathbb{N}=\{n+k \mid k \in \mathbb{N}\}$ where $n \in \mathbb{N}$. Further, $m+\mathbb{N} \subset n+\mathbb{N}$ if and only if $m>n$, for every $m, n \in \mathbb{N}$. Then $S$ is a uniserial $S$-act and the graph $\operatorname{Im} \mathbb{C}(S)$ is complete with countably infinite vertices.

(iii) The cycle $C_{4}$ is the inclusion graph of no $S$-act. Indeed, suppose that $C_{4}$ is the inclusion graph of an $S$-act $A$ and $B_{1}, B_{2}, B_{3}$ and $B_{4}$ are all non-trivial subacts of $A$ as the following:

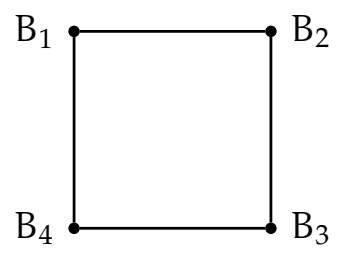

With no loss of generality, assume that $B_{1} \subset B_{2}$. Then $B_{3} \subset B_{2}, B_{3} \subset B_{4}, B_{1} \subset B_{4}$. It is easily seen that $B_{1} \cup B_{3} \neq A, B_{i}$ for all $i \in\{1,2,3,4\}$ which is a contradiction.

(iv) Let $A=\{a, b\}$ be an $S$-act with trivial action. Then $B_{1}=\{a\}$ and $B_{2}=\{b\}$ are only non-trivial subacts of $A$ which are not adjacent and $\operatorname{sog} \operatorname{girth}(\operatorname{Imc}(A))=\infty$.

Theorem 2.3. For each $S$-act $A$, $\operatorname{girth}(\operatorname{Im} \mathbb{C}(A)) \in\{3,6, \infty\}$.

Proof. First we show that for each $n>6$, $\operatorname{girth}(\operatorname{Imc}(A)) \neq n$. On the contrary, let $B_{1}-B_{2}-\cdots-B_{n}-B_{1}$ be the shortest cycle of order $n$. If $B_{1} \cup B_{4} \neq A$, then $B_{1}-B_{1} \cup B_{4}-B_{4}$ is a path with shorter length between $B_{1}$ and $B_{4}$ which is a contradiction. So $B_{1} \cup B_{4}=A$, and by the same way, $B_{1} \cap B_{4}=\emptyset, B_{1} \cup B_{5}=A$ and $B_{1} \cap B_{5}=\emptyset$. Hence, $B_{4}=B_{5}$ which is a contradiction. It remains to show that $\operatorname{girth}(\operatorname{Im} \mathbb{C}(A)) \neq 4,5$. Let $B_{1}-B_{2}-B_{3}-B_{4}-B_{1}$, where $B_{1} \subset B_{2}$, be the shortest cycle in $\operatorname{Im} \mathbb{C}(A)$. Then $B_{3} \subset B_{2}, B_{3} \subset B_{4}$ and 
$B_{1} \subset B_{4}$. It is easily seen that $B_{1} \cup B_{3} \neq A, B_{i}$ for all $i \in\{1,2,3,4\}$. Then $B_{1}-B_{1} \cup B_{3}-B_{2}-B_{1}$ forms a cycle of order 3 which is a contradiction. If $B_{1}-B_{2}-B_{3}-B_{4}-B_{5}-B_{1}$, where $B_{1} \subset B_{2}$, is the shortest cycle in $\operatorname{Im} \mathbb{C}(A)$, then $B_{3} \subset B_{2}, B_{3} \subset B_{4}, B_{5} \subset B_{4}$ and $B_{5} \subset B_{1}$. This implies that $B_{5}$ is adjacent to $B_{2}$ which is a contradiction.

In light of Remark 2.1, Example 2.2 and Theorem 2.3, those cycles which are the inclusion graphs are fully characterized.

Corollary 2.4. The cycle $\mathrm{C}_{\mathrm{n}}$ is the inclusion graph of an $\mathrm{S}$-act if and only if $\mathrm{n}=3$ or $\mathrm{n}=6$.

In the following, we study the connectivity and diameter of the inclusion graphs.

Theorem 2.5. Let $A$ be an $S$-act. Then $\operatorname{Imc}(A)$ is disconnected if and only if it is a null graph with $|\operatorname{Im} \mathbb{C}(A)|=2$. Moreover, if $\operatorname{Imc}(A)$ is connected, then $\operatorname{diam}(\operatorname{Imc}(A)) \leqslant 3$.

Proof. Suppose that $|\operatorname{In} \mathbb{C}(A)| \geqslant 3$. We show that there exists a path between $B_{1}, B_{2}$ for every two distinct non-trivial subacts $B_{1}, B_{2}$ of $A$. Let $B_{1}$ and $B_{2}$ be non-adjacent. If $B_{1} \cap B_{2} \neq \emptyset$ or $B_{1} \cup B_{2} \neq A$, then there exists a $B_{1}, B_{2}$-path. Now let $B_{1} \cap B_{2}=\emptyset$ and $B_{1} \cup B_{2}=A$. Since $|\operatorname{Im} \mathbb{C}(A)| \geqslant 3$, $A$ contains a non-trivial subact $B_{3}$ with $B_{3} \neq B_{1}, B_{2}$. If $B_{1} \cap B_{3}=\emptyset$ and $B_{1} \cup B_{3}=A$, then $B_{2}=B_{3}$ which is a contradiction. So either $B_{1} \cap B_{3} \neq \emptyset$ or $B_{1} \cup B_{3} \neq A$. In the same way, either $B_{2} \cap B_{3} \neq \emptyset$ or $B_{2} \cup B_{3} \neq A$. We consider the following cases:

Case 1. Let $B_{1} \cap B_{3} \neq \emptyset$ and $B_{2} \cap B_{3} \neq \emptyset$. Note that $B_{1} \cap B_{3} \neq B_{2}, B_{2} \cap B_{3} \neq B_{1}$. Then

$$
\mathrm{B}_{1}-\mathrm{B}_{1} \cap \mathrm{B}_{3}-\mathrm{B}_{3}-\mathrm{B}_{2} \cap \mathrm{B}_{3}-\mathrm{B}_{2},
$$

is a $B_{1}, B_{2}$-path provided that $B_{1} \cap B_{3} \neq B_{1}, B_{3}$ and $B_{2} \cap B_{3} \neq B_{2}, B_{3}$. Otherwise, we get a path with shorter length between $B_{1}, B_{2}$. Hence, $d\left(B_{1}, B_{2}\right) \leqslant 4$.

Case 2. Let $B_{1} \cap B_{3} \neq \emptyset$ and $B_{2} \cup B_{3} \neq A$. We have $B_{1} \cap B_{3} \neq B_{2}, B_{2} \cup B_{3} \neq B_{1}$. Then

$$
\mathrm{B}_{1}-\mathrm{B}_{1} \cap \mathrm{B}_{3}-\mathrm{B}_{3}-\mathrm{B}_{2} \cup \mathrm{B}_{3}-\mathrm{B}_{2},
$$

is a $B_{1}, B_{2}$-path provided that $B_{1} \cap B_{3} \neq B_{1}, B_{3}$ and $B_{2} \cup B_{3} \neq B_{2}, B_{3}$. Otherwise, we get a path with shorter length between $B_{1}, B_{2}$. Hence, $d\left(B_{1}, B_{2}\right) \leqslant 4$.

Other cases have the same proof.

The converse is obvious. For the second part, first note that the above proof implicitly states that if $\operatorname{Im} \mathbb{C}(A)$ is connected, then $\operatorname{diam}(\operatorname{Im} \mathbb{C}(A)) \leqslant 4$. We claim that 4 is impossible for the diameter. Assume on the contrary that $\operatorname{Imc}(A)$ is a connected inclusion graph of an $S$-act $A$ with $\operatorname{diam}(\operatorname{Im} \mathbb{C}(A))=4$. Then there exist two distinct vertices $B_{1}, B_{5}$ in $\operatorname{Im} \mathbb{C}(A)$ for which $B_{1}-B_{2}-B_{3}-B_{4}-B_{5}$ is the shortest $B_{1}, B_{5}$-path. It is clear to see that $B_{1} \cup B_{4}=A, B_{1} \cap B_{4}=\emptyset, B_{1} \cup B_{5}=A$ and $B_{1} \cap B_{5}=\emptyset$. Thus we get $B_{4}=B_{5}$ which is a contradiction.

Corollary 2.6. Let $A$ be an $S$-act with two zero elements and $|A| \geqslant 3$. Then $\operatorname{Im} \mathbb{C}(A)$ is connected.

Proof. If $\theta_{1}$ and $\theta_{2}$ are two zero elements of $A$, then the sets $\left\{\theta_{1}\right\},\left\{\theta_{2}\right\}$ and $\left\{\theta_{1}, \theta_{2}\right\}$ are distinct non-trivial subacts of $A$. Hence, by Theorem $2.5, \operatorname{Im} \mathbb{C}(A)$ is connected.

In what follows, we study the connectivity of the inclusion graphs of cyclic, free and cofree S-acts. Let us first recall some definitions from [10].

By a cyclic $S$-act, we mean an $S$-act $A$ generated by an element $a \in A$, that is, $A=S a$ where

$$
\mathrm{Sa}=\{\mathrm{sa} \mid \mathrm{s} \in \mathrm{S}\} .
$$

An $S$-act $A$ is called free if it has a basis $X$, i.e., each element $a \in A$ is uniquely represented as $a=s x$ for some $s \in S$ and $x \in X$. In this case, $A \cong \bigsqcup_{x \in X} S$. The dual categorical notion of free is the cofree $S$-act which is isomorphic to an $S$-act of the form $X^{S}$, the set of all maps from $S$ to a non-empty set $X$, with the action given by $(s f)(t)=f(t s)$ for $s, t \in S$ and $f \in X^{S}$. The set $X$ is called a cobasis for $A$. 
Proposition 2.7. Let A be an S-act. Then the following assertions hold:

(i) If $A$ is cyclic, then $\operatorname{Im} \mathbb{C}(A)$ is connected. In particular, $\operatorname{Im} \mathbb{C}(S)$ is connected.

(ii) If $A$ is a free $S$-act with a basis $X$ where $|X|>2$, then $\operatorname{Im} \mathbb{C}(A)$ is connected.

(ii) If $A$ is a cofree $S$-act and $|A| \geqslant 3$, then $\operatorname{Im} \mathbb{C}(A)$ is connected.

Proof.

(i) Consider a cyclic $S$-act $A$ with disconnected inclusion graph. Using Theorem 2.5, $A$ has only two non-trivial subacts, say $B$ and $C$, such that $B \cup C=A$ and $B \cap C=\emptyset$. Clearly, $B$ and $C$ are simple subacts of $A$ so that $A$ is completely reducible. Note that a cyclic $S$-act is completely reducible if and only if it is simple (see [10, Lemma I.5.32]). This implies that $A$ is simple which is a contradiction.

(ii) It follows from hypothesis that the number of non-trivial subacts of $A$ is greater than 2 . Hence, Theorem 2.5 gives the assertion.

(iii) Using the assumption, $A$ can be considered as the $S$-act $X^{S}$ for a cobasis $X$ where $|X|>1$. Since every constant map in $A$ is a zero element and there exist exactly $|X|$ constant maps in $A, A$ contains at least two zero elements and hence $\operatorname{Im} \mathbb{C}(A)$ is connected by Corollary 2.6.

A non-trivial subact $M$ of an $S$-act $A$ is called minimal, if $B \subseteq M$ for some subact $B$ of $A$ implies that $B=M$. We denote the set of all minimal subacts of $A$ by $\operatorname{Min}(A)$.

Remark 2.8. Let $A$ be an $S$-act. If $\operatorname{deg}(M)<\infty$ for a minimal subact $M$ of $A$, then the number of minimal subacts of $A$ is finite. Indeed, if $M_{1}, M_{2}, M_{3}, \cdots$ be infinite minimal subacts of $A$ other than $M$, then the infinite strict ascending chain

$$
M \subset M \cup M_{1} \subset M \cup M_{1} \cup M_{2} \subset \cdots,
$$

gives that $\operatorname{deg}(M)=\infty$ which is a contradiction. Further, if $\operatorname{Im} \mathbb{C}(A)$ is complete, then $A$ contains at most one minimal subact.

Theorem 2.9. Let $\mathrm{A}$ be an $\mathrm{S}$-act and $\operatorname{Im\mathbb {C}}(\mathrm{A})$ have no cycle. Then $\operatorname{Im} \mathbb{C}(\mathrm{A})$ is a null graph (with one or two vertices) or $\mathrm{P}_{i}$ where $\mathrm{i} \in\{1,2,3,4\}$.

Proof. It follows from the assumption that $A$ has a minimal subact. If $M_{1}, M_{2}, M_{3}$ are three distinct minimal subacts of $A$, then

$$
M_{1}-M_{1} \cup M_{2}-M_{2}-M_{2} \cup M_{3}-M_{3}-M_{3} \cup M_{1}-M_{1},
$$

is a cycle which is a contradiction. So $|\operatorname{Min}(A)| \leqslant 2$. The following cases may occur.

Case 1 . Let $A$ have only one minimal subact, say $M$. Then every subact of $A$ contains $M$. We claim that $|\operatorname{Im} \mathbb{C}(A)| \leqslant 3$. On the contrary, let $B_{1}, B_{2}, B_{3}$ be another distinct non-trivial subacts of $A$. Since $\operatorname{Im} \mathbb{C}(A)$ has no cycle, $B_{1} \cup B_{3}=B_{2} \cup B_{3}=A$ and $B_{1} \cap B_{3}=B_{2} \cap B_{3}=M$ whence $B_{1}=B_{2}$ which is a contradiction. Thus the graph $\operatorname{Im} \mathbb{C}(A)$ is one of the graphs: one-vertice graph, or the paths $P_{1}$ or $P_{2}$.

Case 2. Let $A$ have two distinct minimal subacts, say $M_{1}, M_{2}$. If $M_{1} \cup M_{2}=A$, then $A$ has no another non-trivial subact and $\operatorname{Im} \mathbb{C}(A)$ is a null graph with two distinct vertices. If $M_{1} \cup M_{2} \neq A$, then $\operatorname{Im} \mathbb{C}(A)$ contains at least the three vertices $M_{1}, M_{2}, M_{1} \cup M_{2}$. we claim that $|\mathbb{I m} \mathbb{C}(A)| \leqslant 5$. Assume contrarily that $B_{1}, B_{2}, B_{3}$ are another distinct non-trivial subacts of $A$. We show that each $B_{i}$ contains only one minimal. Otherwise, $M_{1} \cup M_{2} \subset B_{i}$ and then $M_{1}-M_{1} \cup M_{2}-B_{i}-M_{1}$ is a cycle which is a contradiction. Moreover, if $B_{i}$ and $B_{j}$ intersect in a minimal subact as $M_{1}$, then $B_{i} \cup B_{j} \neq A$ because $M_{2} \nsubseteq B_{i} \cup B_{j}$ and in this case the cycle $M_{1}-B_{i}-B_{i} \cup B_{j}-M_{1}$ yields a contradiction. Therefore, each $B_{i}$ contains only one minimal subact and each minimal subact is contained in only one $B_{i}$. This contradicts the number of $B_{i}$ 's. So, in addition to $M_{1}, M_{2}, M_{1} \cup M_{2}, \operatorname{Im} \mathbb{C}(A)$ contains at most two another vertices. It is straightforward to see that $\operatorname{Im} \mathbb{C}(A)$ is one of the paths $\mathrm{P}_{2}$ or $\mathrm{P}_{3}$ or $\mathrm{P}_{4}$. 
Here we study the domination number of the inclusion graphs and determine them for the graphs of some $\mathrm{S}$-acts.

Let $\mathrm{G}$ be a graph. The (open) neighborhood $N(x)$ of a vertex $x \in V(G)$ is the set of vertices which are adjacent to $x$. For a subset $T$ of vertices, we put

$$
N(T)=\bigcup_{x \in T} N(x), \quad N[T]=N(T) \cup T .
$$

A set of vertices $T$ in $G$ is a dominating set, if $N[T]=V(G)$. The domination number of $G$ is the minimum cardinality of a dominating set of $\mathrm{G}$ and is denoted as $\gamma(\mathrm{G})$.

An $S$-act $A$ is said to be Artinian, if every descending chain of subacts of $A$ terminates. It can be easily seen that every non-empty subact of an Artinian S-act contains a minimal subact.

Proposition 2.10. Let $A$ be an S-act. Then $\gamma(\operatorname{Imc}(A)) \leqslant 2$ provided that each of the following assertions hold:

(i) A contains a minimal subact;

(ii) A contains a zero element;

(iii) $|\operatorname{Sub}(A)|<\infty$;

(iv) $|A|<\infty$;

(v) A has trivial action;

(vi) A is Artinian.

Proof.

(i) Let $M$ be a minimal subact of $A$ and $W:=\{B \in \operatorname{Sub}(A) \mid M \nsubseteq B\}$. If $W=\emptyset$, then for every non-trivial subact $B$ of $A, M \subseteq B$ and so $\{M\}$ is a dominating set. If $W \neq \emptyset$, then $\left\{M, \bigcup_{B \in W} B\right\}$ forms a dominating set in $\operatorname{Im} \mathbb{C}(A)$. Hence, $\gamma(\operatorname{Im} \mathbb{C}(A)) \leqslant 2$.

(ii) Using (i), $\{z\}$ is a minimal subact of $A$ where $z$ is a zero element.

The assertions (iii),(iv),(v) and (vi) are consequences of (i).

Proposition 2.11. The following assertions hold:

(i) Let $A$ be the coproduct of a family $\left\{A_{i} \mid i \in I\right\}$ of $S$-acts with $|\mathrm{I}|>1$ and $\gamma\left(\operatorname{Im} \mathbb{C}\left(A_{j}\right)\right)=1$ for some $j \in \operatorname{I}$. Then $\gamma(\operatorname{In} \mathbb{C}(A))=2$.

(ii) If $\mathrm{F}$ is a free $\mathrm{S}$-act with a non-singleton basis and $\gamma(\operatorname{Im} \mathbb{C}(\mathrm{S}))=1$, then $\gamma(\operatorname{Im} \mathbb{C}(\mathrm{F}))=2$.

Proof.

(i) Suppose that $\{T\}$ is a dominating set of $\operatorname{Imc}\left(A_{j}\right)$. Let $B=\bigsqcup_{i \in I} B_{i}$ be a non-trivial subact of $A$ where $B_{i}$ 's are (possibly empty) subacts of $A_{i}$. If $B_{j} \subseteq T$, then $B \subseteq \bigsqcup_{i \in I} U_{i}$ where $U_{j}=T, U_{i}=A_{i}$ for all $i \neq j$ and if $T \subseteq B_{j}$, then $T \subseteq B$. Thus $\left\{\coprod_{i \in I} U_{i}, T\right\}$ is a dominating set of $\operatorname{Im} \mathbb{C}(A)$ so that $\gamma(\operatorname{Im} \mathbb{C}(A)) \leqslant 2$. Now we show that $\gamma(\operatorname{Im} \mathbb{C}(A)) \neq 1$. On the contrary, let $\left\{B=\bigsqcup_{i \in I} B_{i}\right\}$ be a dominating set of $\operatorname{Im} \mathbb{C}(A)$ and $s \in I$. Then one of the subacts $\coprod_{i \neq s} A_{i}$ or $A_{s}$ is non-adjacent to $B$ in $\operatorname{Im} \mathbb{C}(A)$ which is a contradiction.

(ii) follows from (i).

Example 2.12. Consider the monoid $S=\{1, s\}$ where $s$ is an idempotent element and the $S$-act $A=\{a, b, c\}$ with the action defined by $1 c=c, s c=a$ and $a, b$ are fixed elements. Then all non-trivial subacts of $A$ are the sets $\{a\},\{b\},\{a, b\}$ and $\{a, c\}$. It is clear that $\{\{a\},\{b\}\}$ is a dominating set in $\operatorname{Im} \mathbb{C}(A)$ and $\gamma(\operatorname{Im} \mathbb{C}(A))=2$.

An independent set in a graph is a set of pairwise non-adjacent vertices. The independence number of $\mathrm{G}$, written as $\alpha(\mathrm{G})$, is the maximum size of an independent set.

Remark 2.13. In [13], it has shown that the independence number of the intersection graph of an $S$-act $A$ equals the number of minimal subacts of $A$. But this is not the case for the inclusion graphs. For instance, let $A=\{a, b, c, d\}$ be an $S$-act with trivial action. Then $\alpha(\operatorname{Im} \mathscr{C}(A))=6$ whereas $|\operatorname{Min}(A)|=4$. 


\section{Acknowledgment}

We would like to express our gratitude and appreciation to the referees for commendable comments which improved the text.

\section{References}

[1] S. Akbari, M. Habibi, A. Majidinya, R. Manaviyat, The inclusion ideal graph of rings, Comm. Algebra, 43 (2015), 2457-2465. 1

[2] D. F. Anderson, A. Badawi, The total graph of a commutative ring, J. Algebra, 320 (2008), 2706-2719. 1

[3] J. Bosák, The graphs of semigroups, in: Theory of Graphs and its Application, 1964 (1964), 119-125. 1

[4] B. Csákány, G. Pollák, The graph of subgroups of a finite group, Czechoslovak Math. J., 19 (1969), 241-247. 1

[5] A. Das, Subspace inclusion graph of a vector space, Comm. Algebra, 44 (2016), 4724-4731. 1

[6] A. Delfan, H. Rasouli, A. Tehranian, Intersection graphs associated with semigroup acts, submitted. 1

[7] F. R. DeMeyer, T. McKenzie, K. Schneider, The zero-divisor graph of a commutative semigroup, Semigroup Forum, 65 (2002), 206-214. 1

[8] P. Devi, R. Rajkumar, Inclusion graph of subgroups of a group, Cornell University Library, 2016 (2016), 22 pages. 1

[9] A. A. Estaji, T. Haghdadi, A. A. Estaji, Zero divisor graphs for S-act, Lobachevskii J. Math., 36 (2015), 1-8. 1

[10] M. Kilp, U. Knauer, A. V. Mikhalev, Monoids, Acts and Categories, Walter de Gruyter \& Co., Berlin, (2000). 1, 2, 2

[11] H. R. Maimani, M. R. Pournaki, S. Yassemi, Weakly perfect graphs arising from rings, Glasg. Math. J., 52 (2010), 417-425. 1

[12] R. Nikandish, M. J. Nikmehr, The intersection graph of ideals of $\mathbb{Z}_{n}$ is weakly perfect, Cornell University Library, 2013 (2013), 8 pages. 1

[13] H. Rasouli, A. Tehranian, Intersection graphs of S-acts, Bull. Malays. Math. Sci. Soc., 38 (2015), 1575-1587. 1, 2.13

[14] M. R. Sorouhesh, H. Doostie, C. M. Campbell, A sufficient condition for coinciding the Green graphs of semigroups, J. Math. Computer Sci., 17 (2017), 216-219. 1

[15] D. B. West, Introduction to Graph Theory, Prentice Hall, Upper Saddle River, (2001). 1 